\title{
Research Progress of Fouling Mechanism and Control Technology of Ceramic Membrane
}

\author{
Jingxiu ZHONG ${ }^{1, a}$,Shoubin ZHANG ${ }^{1, b^{*}}$, Huajun $\mathrm{SUN}^{2, c}$ \\ ${ }^{1}$ School of Civil Engineering \& Architecture, University of Jinan, NO.336, Nanxinzhuang West Road., \\ Jinan 250022, P.R.China \\ ${ }^{2}$ Advanced Ceramic Institute, Zibo New \& Hi-tech Industrial Development Zone, Zibo 255000 , \\ P.R.China

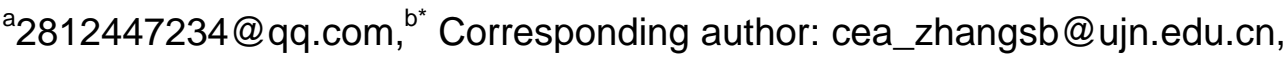 \\ c174051406@qq.com
}

Keywords: ceramic membrane; fouling mechanism ; control technology ;

Abstract. In recent years, inorganic ceramic membranes have been successfully applied in water treatment because of their unique advantages. However, the membrane fouling problems during the application of inorganic ceramic membranes should not be underestimated. With the wide application of inorganic ceramic membrane in water treatment, membrane fouling control technology has attracted more and more attention. Based on the fouling mechanism of ceramic membrane, the cleaning methods and control techniques of membrane fouling are reviewed. It provides some new ideas and methods for the control of ceramic membrane fouling, and also provides a reference for the expanded application of ceramic membrane in water treatment.

\section{Introduction}

With the rapid development of economy and the increase of population, the shortage of water resources is increasingly prominent. The traditional water treatment technology has been unable to meet the current demand of water resources. Therefore, a series of new water treatment technologies have been developed. Among them, membrane separation technology is a high and new technology developed in the last 30 years. It is a cross combination of material science and media separation technology. Furthermore, it is highly valued for its high efficiency, easy operation, compact equipment, no phase change and energy saving ${ }^{[1,2]}$. Among them, inorganic membranes are dominated by ceramic membranes, and have the incomparable advantages of organic membranes. They have partially replaced the organic films in water treatment.Inorganic ceramic membrane has many advantages, such as corrosion resistance, high temperature resistance, stable structure, large mechanical strength and long service life. It has been successfully applied in water treatment ${ }^{[3]}$.

\section{Fouling mechanism of ceramic membrane}

The classic membrane fouling model can be divided into the following four types: complete blockage model, intermediate plugging model, filter cake plugging model and standard plug model $^{[4-8]}$. The complete blocking model assumes that the contaminant particles plug the membrane pores completely and the contaminant particles do not overlap with each other, and a monolayer deposition occurs on the membrane surface; The intermediate blocking model is similar to the fully blocking model. It is assumed that the contaminant particles clog the membrane pores, but allows accumulation or deposition on the membrane surface between contaminant particles; The cake layer model assumes that the particles of the contaminant accumulate on the membrane surface in a superposition manner, and the cake layer is formed on the membrane surface; The standard plugging model assumes that contaminant particles enter the membrane channel and plug in the pores, that is, membrane fouling occurs mainly within the membrane pores rather than the surface ${ }^{[9,10]}$. It is generally believed that standard plug model occurs in the membrane pores, which can cause irreversible holes to block pollution, while complete blockage model, intermediate plugging model 
and filter cake plugging model mainly occurs on the surface of the membrane, and the pollution is often reversible pollution ${ }^{[21-24]}$. Figure 2-1 shows four classical membrane fouling models and their corresponding equations.

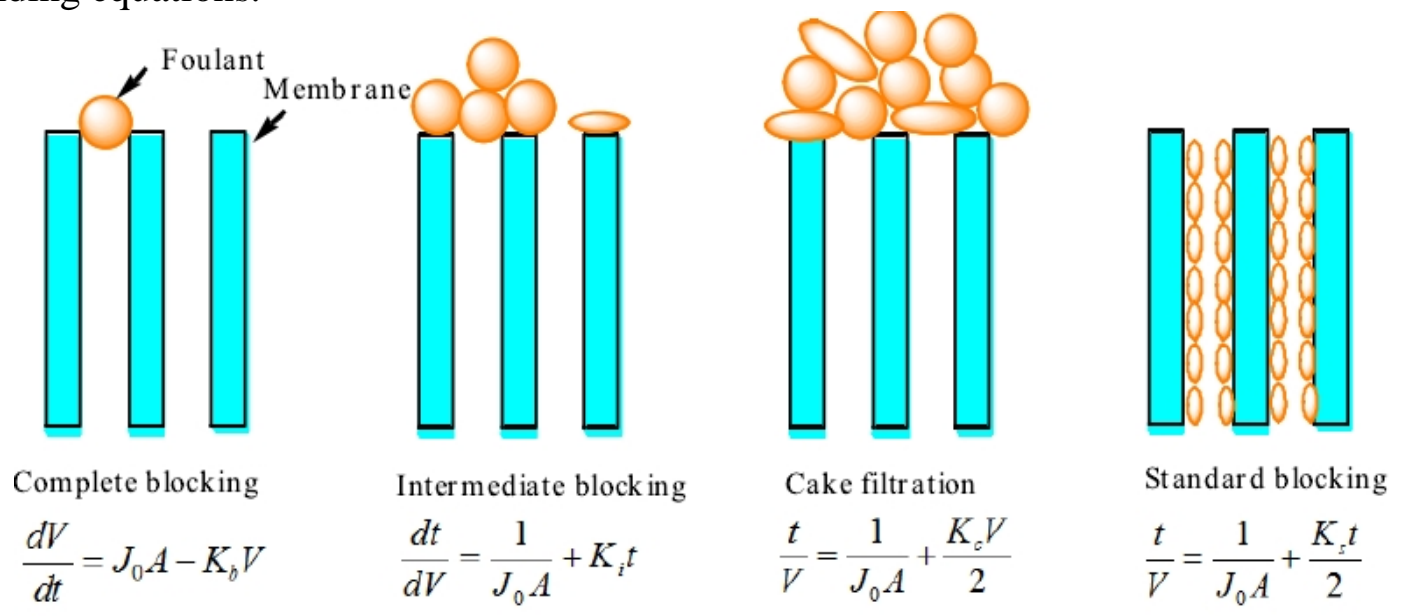

Fig.2-1 Schematic diagrams and equations of four fouling models: A, membrane area; $t$, filtration time; $\mathrm{V}$, cumulative permeate volume; $\mathrm{J}_{0}$, initial permeate flux; $\mathrm{K}_{\mathrm{b}}, \mathrm{K}_{\mathrm{i}}, \mathrm{K}_{\mathrm{c}}$, and $\mathrm{K}_{\mathrm{s}}$, the coefficients of complete blocking, intermediate blocking, cake filtration and standard blocking

\section{Techniques of ceramic membrane pollution cleaning and control}

\section{Ceramic membrane pollution cleaning method}

Physical cleaning Physical cleaning methods are mainly mechanical cleaning, including forward washing, directional cleaning, through liquid backwashing, exhaust water flushing, automatic sponge ball cleaning, ultrasonic cleaning, electric field filtration and cycle washing and so on. The physical cleaning method has the characteristics of not introducing new pollutants, simple cleaning steps and on-line cleaning, but the method is effective only in the early stage of fouling of ceramic membrane, and the cleaning effect decreases rapidly with time. Physical cleaning is not specifically targeted, generally able to remove a variety of pollutants, but not clear enough ${ }^{[11]}$. The chemical cleaning method is to clean the water by adding a specific chemical agent. This method can remove the composite fouling and recover the flux quickly.

Chemical cleaning Chemical cleaning is to remove sediment and dirt from the membrane by using chemical reagents. Frequently-used chemical cleaning agents are acids, bases, oxidants and chelating agents and so on. Chemical cleaning has the possibility of introducing new pollutants into the membrane system. At the same time, the possibility of chemical action between pollutants and membranes can not be ruled out, and there are many conversion steps between operation and cleaning, and they must be washed off-line. Different pollution systems are very different. Therefore, it is necessary to select and distinguish the cleaning methods and cleaning agents according to the physicochemical properties of the pollutants on the surface of the membrane, so as to clean them in a targeted way $^{[12]}$.

Specific cleaning methods should be adopted for specific contaminants. For instance, the inner surface of the hollow fiber membrane, which is easy to breed microbes, is washed with acid, and the effect of improving membrane specific flux is obvious; At present, new control methods such as ultrasonic on-line cleaning have also been widely used; The latter is adopted when the former method is difficult to achieve the desired effect, such as the removal of biological pollution and organic pollution. Oxidants are mainly used while the removal of inorganic scale requires acid cleaning agent. In order to enhance the removal effect, cleaning agents are used in combination. However, there is a certain degree of corrosion of these cleaning agents. Therefore, the rationality of the selection should be guaranteed so as not to cause damage to the surface of the film ${ }^{[13]}$. 


\section{Membrane fouling control technology}

Improved coagulation pretreatment method Coagulation pretreatment usually refers to adding flocculant to flocculation removal of pollutants in water, reducing suspended substances in water to reduce membrane fouling. However, there are many problems such as increasing energy consumption and producing by-products. Electrical coagulation can avoid these problems.

$\mathrm{KIM}$ et $\mathrm{a}^{[14]}$ uses flocculation and flocculation $(\mathrm{CF})$ pretreatment to solve the membrane fouling in the process of oil sand wastewater (OSPW) membrane treatment. The results show that $\mathrm{CF}$ pretreatment can effectively improve the membrane flux of nanofiltration (NF) and (FO), and the types of coagulant and coagulant will influence the pollution index; In the coagulant aid, the anionic assistant agent improved the membrane electronegativity and reduced the formation of the fouling layer, thus increasing the membrane flux to the greatest extent; On the contrary, although the cationic coagulant reduced the turbidity of OSPW effectively, it aggravated membrane fouling. Some scholars ${ }^{[15]}$ have studied the removal of colloidal organic compounds and their inhibitory effects on membrane fouling by coagulation and magnetic ion exchange resin (MIEX). It is found that flocculation can not remove colloidal organic matter selectively, and the optimum amount of removal depends on the amount of humic acid in organic matter; while MIEX can remove humic substances preferentially and reduce the amount of coagulant needed to remove colloidal organic matter. Therefore, when the dosage of coagulant is low, the method of combining coagulant with MIEX is consistent with using coagulant alone for the effect of anti pollution. The study confirmed that the combination of coagulant and MIEX can reduce membrane fouling effectively. This method also solves the problem of high cost and reproducibility when using MIEX alone.

Nano modification of membrane surface In the process of membrane filtration, organic pollution and biological pollution are often the two major factors causing irreversible fouling and membrane flux decline. Past research has generally focused on the control of single pollution ${ }^{[16]}$. Some scholars reduce organic pollution and biological pollution simultaneously by using polyelectrolyte and nano silver to modify the MF film simultaneously. The results showed that both membrane flux and membrane resistance decreased after modification.Ho wever, its pollution resistance has been significantly improved. After silver modification, the growth of the film surface was almost zero, mainly because the silver ions released by the nano silver inhibited the growth of microorganisms. The method realizes inhibition of organic pollution and biological pollution simultaneously.

\section{Conclusions}

From the above review, we can see that there have been many breakthroughs in the study of fouling of ceramic membranes at home and abroad in recent years. There is a better understanding of the fouling mechanism of ceramic membranes, and a more thorough study has been done on membrane fouling, cleaning and control techniques. These studies will provide some new ideas and methods for ceramic membrane fouling control, and also provide a basis for the wider application of ceramic membrane in water treatment.

With the continuous progress of science and technology and increasing attention to environmental protection issues around the world, inorganic ceramic membranes are more and more widely used in wastewater treatment and other fields because of their advantages of corrosion resistance, high temperature resistance, structural stability, mechanical strength and long service life, and the porous ceramic material industry will also usher in a broader development prospects.

\section{Acknowledgements}

This work was financially supported by the Shandong Provincial Key Research and Development Program(2016CYJS07A03-2), Shandong Provincial Natural Science Foundation, China (ZR2015EM021), Doctoral Foundation of University of Jinan (XBS1432) and partly supported by National Natural Science Foundation of China (51678276). 


\section{References}

[1] Tian Yuelin, Liu guizhong, Yang Yongqiang, et al. Research on Inorganic Membrane Separation Technology Applicationin the Field of Water Treatment[J]. Environmental Protection Science. Vol. 37(2011), p.16-19.

[2] YAO Jilun, ZHANG Xing, ZHOU Zhen, et al. Review of Ceramic Membrane in Water Treatment[J]. Journal of Chongqing University of Technology(Natural Science). Vol. 30(2016), p. 69-74.

[3] Wang Yi, Xia Shengji. Syenthesis and Application on of Ceramic Membrane for Water Treament[J]. Water Purification Technology. Vol. 43(2017), p. 107.

[4] Huang H, Young T A, Jacangelo J G. Unified membrane fouling index for low pressure membrane filtration of natural waters: principles and methodology[J]. Environmenal Science \& Technology. Vol. 42(2007), p.714-720.

[5] M.A. Green: Study on the difference and mechanism of MIEX and PAC membrane fouling in integrated process (Trans Tech Publications, Tianjin 2016).

[6] M.A. Green: Fouling mechanism and antifouling modification of ceramic

Ultrafiltration membrane for treating O/W emulsion[D]. (Harbin Institute of Technology, Harbin 2016).

[7] Zhou J E, Chang Q B, Wang Y Q, et al. Separation of Stable Oil-water Emulsion by the Hydrophilic Nano-sized $\mathrm{ZrO} 2$ Modified Al2O3 Microfiltration Membrane[J]. Separation and Purification Technology. Vol. 75( 2010), p. 243-248.

[8] Hermia J. Constant Pressure Blocking Filtration Law Application to Power-Law Non-Newtonian Fluid[J]. Transactions of the institution of Chemical Engineers. Vol. 60(1982)p.183-187.

[9] Duclos-Orsello C, Li W, Ho C-C. A Three Mechanism Model to Describe Fouling of Microfiltration Membranes[J]. Journal of Membrane Science. Vol. 280(2006), p.856-866.

[10] Costa A R, De Pinho M N, Elimelech M. Mechanisms of Colloidal Natural Organic Matter Fouling in Ultrafiltration[J]. Journal of Membrane Science. Vol. 281(2006), p.716-725

[11] XU Yiqing, YANG Hui, LUO Youhua. Membrane cleaning progress optimization of ceramic membrane microfiltration of the compound banlangen liyan granules water extraction liquid. Strait Pharmaceutical Journal. Vol. 28( 2016), p.27-31.

[12] FU Wanxia, LI Lei. Study on membrane cleaning methods and mechanisms in membrane bioreactor[J]. Technology and equipment for environmental pollution control. Vol. 12(2016), p.20-25.

[13] ZHANG Chuan, LIU Xinyang, WANG Yi, et al. Performance of substrate transport and bio-degradation within annular optical-fiber-illuminating biofilm reactor during continuous photo-H2 production. Journal of agricultural machinery. Vol. 46(2015), p.175-179.

[14] KIM E S, LIU Y, GAMAL EL DIN M. Evaluation of membrane fouling for in-line filtration of oil sands process-affected water: the effects of pretreatment conditions[J]. Environmental Science \& Technology. Vol. 46(2012), p.2877-2884.

[15] HUANG H, CHO H H, JACANGELO J G, et al. Mechanisms of membrane fouling control by intergrated magnetic ion exchange and coagulation[J]. Environmental Science \& Technology. Vol. 46(2012), p.10711-10717.

[16] Xue Yonggang, Guan Song, Dai Xiaohu, et al. New prograss in membrane fouling mechanism and its control technique[J]. Environmental pollution and control. Vol. 36( 2014), p.74-78. 